\title{
Research on Black Box Testing Technology Based on Virtual Reality
}

\author{
Song Tang \\ Hunan Mass Media Technology College
}

Keywords: Black box testing, Virtual reality, VRML

\begin{abstract}
This paper is focused on the concept of virtual testing technology based on VR, putting its focus on the systematic study of virtual testing technology based on VR from a macro perspective, so as to establish the basic concept and theoretical model as well as implementation methods, the specific content can be divided into three parts: theoretical basis, technology of realization, examples of application. Thus the application of virtual reality technology to black box testing is studied systematically in this paper.
\end{abstract}

\section{Introduction}

VR technology can provide real-time interactive operation, 3D visual space and interface with multi channels for users (visual, auditory and tactile), which can simulate the real world, the dynamic environment can make the real-time response to the user's posture and language command. VR technology can change the way that people obtain information, so as to improve the harmony between human and machine and make the human-computer interface more intuitive[1]. VR's immersive and interactive features will play a very important role in improving the testing environment.

In this context, combined with the development of VR technology, simulation technology and the inherent demand of testing technology[2], it makes a systematic research on the theory and application foundation of virtual testing technology based on VR in this paper. Virtual testing technology based on VR is a new technology for testing that is formed by VR simulation technology, which can make it possible that generated by computer software on the simulation and testing of product performance in the virtual test environment. In this paper, virtual reality software black box testing is incorporated into the architecture of Virtual Manufacturing(VM), proposing that black box testing should be put into the whole lifecycle of VM[3]. It not only can provide a clear application background for virtual testing technology based on VR, but also can extend the meaning of VM to make it more widely oriented to manufacturing objects and processes. VM can provide the discipline background for virtual testing technology, which can also extend the application scope of virtual testing technology[4].

This paper is based on the previous work that is related to virtual testing technology theory model based on VR, system structure, methodology foundation, theoretical basis and key realization techniques, which make a comprehensive exploratory research. Thus it can further improve the theory of virtual testing technology based on VR. At the same time, through the exploratory research on the implementation of virtual test system based on VR, it lays a foundation for the practical application of virtual testing technology based on VR. 


\section{The Concept of Black Box Testing}

Black box testing is also called functional testing, data-driven testing, or testing based on specification. It is a test from the user's point of view. The tester treats the test program as a black box. It is a function that a given product should have, through tests we can see whether each function is functioning properly or not. During the period of testing,without considering the internal structure and internal characteristics of the program, the tester can test the program at the port of the interface. It can only check whether the program function is normally used by the requirements of the specification: whether the program can receive the input data properly and produce the correct output information, so as to maintain the integrity of the external information. The main methods of black box testing include equivalence class partition, boundary value analysis, causality diagram, and error inference and so on, which are mainly used in software verification test. The "Black Box" approach puts its focus on the external structure of program, without considering the internal logic structure, it can test according to the software interface as well as the function of software.

\section{Nondestructive Testing System of Black Box Based on Web}

The requirement of different types of VR systems on hardware is quite different, and the cost of them varied greatly. As for the method of displaying, VR system can be divided into two broad categories: wide screen projection system and desktop system. The former is large in scale, large in investment, which is suitable for multi person sharing or special simulation systems. The latter is small and compact, which is suitable for single use as well as promotion. So far desktop system has been widely used in a variety of research areas, such as simulation, bimolecular structure simulation, geological faults, meteorological distribution[5], GIS and so on. In fact, the main obstacle to the current VR system's practicality is cost. During the period of application, the VR system should choose the system with high performance price ratio according to the specific requirements of the project and funds. The desktop VR system although I dark field is poor, but because of its low cost, its application is still relatively wide. In addition, due to the current performance and workstation PC machine distance has been greatly reduced, the multimedia technology based on PC has been developed rapidly, which can make it become the mainstream of application system with the application of VR in PC. Thus it can provide an opportunity for VR to spread across the PC platform. Especially in China, the development of VR application to PC platform is more valuable. While Europe and America as well as other countries are developing immersive VR, there are also a large number of VR systems based on PC platform.

In this paper, the hardware platform adopts desktop VR system (Desktop VR), the main considerations are as follows:

1. A realistic VR system requires tracking system, vision system, haptic system, audio system, image generation system, and display devices. According to the characteristics and actual needs of the project, we should put focus on the simulation and results of NDT process. Moreover, we should ignore the effects of touch and audio for the time being.

2. Desktop VR system, generally we use PC and low type workstation to realize simulation. In fact, with the rapid development of computer technology, the performance of high type PC machines has been almost the same as the performance of middle type workstation. Equipped 
with stereoscopic shutter glasses and simple 3D mouse, a basic Desktop VR system can be formed. For the common researching designer, it's easy to construct.

3. By using three-dimensional shutter glasses, it can make the observer produce a three-dimensional visual effect. This effect can be achieved by adopting Deshop VR system. In order to implement the virtual testing environment, a suitable VR modeling tool is also needed. In this paper, the current popular VRML (Virtual Reality, Modeling Language, namely, VR modeling language) is used as three-dimensional development tool. VRML is a standard for describing scenarios in a virtual environment. It defines the language descriptions commonly used in 3D applications, such as hierarchical transformation, light sources, viewpoint, geometry, animation, fog, material features and texture mapping and so on, which has simple description behavioral features. Therefore, it is an indispensable tool language for the construction of desktop VR system.

\section{Execution Mode of VRML}

By using Script node programming of VRML, accessing events between Java and setting up the internal message channel of scene map, it can realize the interaction and animation function of the virtual entity easily. As you can see from Fig.1, VRML interaction and animation execution are driven by the event. VRML scenario can accept two event drivers: the events passing from routing statements and direct events written by the external program interfaces. The routing statement can describe the delivery path of each message that is passing from the scene, i.e., the event is passed from the domain of EventOut from a certain node to the domain of EventIn of a certain nod. In the scene, the sensor node usually can define the triggering event, which is routed through routes to EventIn of other nodes in the scene graph. If the trigger events of sensor nodes are transmitted directly to the interpolator nodes so as to produce the key value of interpolation, which can also be sent to the Script nodes for processing and generating key value of interpolation. The process of dealing Script node is a scripting program written in JavaSeript syntax. The Script node can also prevent event processing by introducing Java program (i.e., Class file) through URL domain introducing Java program. The result after the node responding to the event can continue passing route data to other nodes that needed, such as transferring to the entity, so as to change its location and shape. The direct event written by the external program interface does not require a routing diagram to pass, but the other execution processes are the same. If you need an external program's response, it it needs another program to respond, it should have the interface to read the data out of the EventOut. 


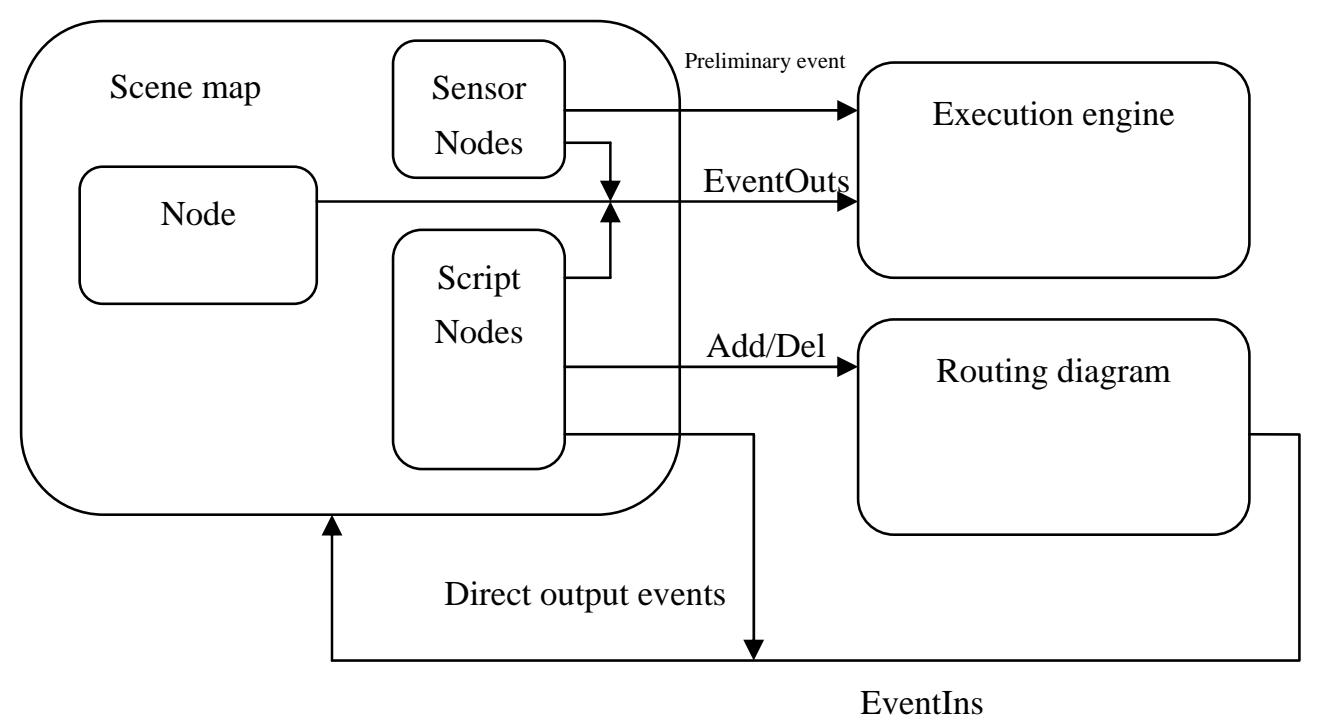

Fig.1. Event Flow Diagram in Scenario Diagram Event System

\section{Conclusion}

Combining VR technology, simulation technology and black box testing technology together to form virtual test technology based on VR, which can undoubtedly have the innovative significance and practicability. But since the virtual test technology based on VR is a relatively new concept, the related involved theory and technology are quite diverse. The scope of application is quite broad. Moreover, restricted by the level of ability and objective conditions, the research content of this article is only preliminary research and discussion. In the theoretical system, it needs further improvement and clarification.

The way of realizing the interactive function of human-computer interaction of VRML in VRML is very powerful, in VR simulation, it can make good use of the function of the system, as for the generated events by user generated by users can make response according to the definition of "good behavior".

Sensors (or detectors) are primitives that provide interactive capabilities and dynamic behavior in VRML. A node with dynamic capability needs to contain sensor. VRML can provide seven sensor nodes, namely, Cylinder Sensor, Plane Sensor, Time Sensor, Touch Sensor, Visibility Sensor, Proximity Sensor. They can provide a mechanism for users to interact with objects in the virtual world: according to the clock or the user's actions, they can produce the corresponding event, this event cam pass down along the predetermined route, which can make the virtual world respond to users, so as to realize the interaction. For example, Touch Sensor can be used to respond to user actions (clicking mouse), Proximity Sensor can be used to detect the user in a defined area within the movement, Collision node can be used to detect the collision and the conflict of the objects and so on.

Because the embedded scripting language can only carry on simple operation, so as for the complicated operation, it needs the help of external programming interface, by using JAVA language to write the corresponding response function, so as to complete the necessary interactive computing, then through the scene, the routing graph event can be passed to the related nodes to complete the cycle of one event, as well as the interaction with users. 


\section{References}

[1] Cates C U, Lönn L, Gallagher A G. Prospective, randomised and blinded comparison of proficiency-based progression full-physics virtual reality simulator training versus invasive vascular experience for learning carotid artery angiography by very experienced operators[J]. BMJ Simulation and Technology Enhanced Learning, 2016, 2(1): 1-5.

[2] Yuhua S. Research on the Application of the Culture Resource Management Based on Big Data Technology[J]. Journal of Applied Science and Engineering Innovation, 2017, 4(2): 64-68.

[3] Ravi D K, Kumar N, Singhi P. Effectiveness of virtual reality rehabilitation for children and adolescents with cerebral palsy: an updated evidence-based systematic review[J]. Physiotherapy, 2017, 103(3): 245-258.

[4] Yang J. Research on a Improve of Bat Algorithm in the Cloud Computing Resources[J]. Journal of Applied Science and Engineering Innovation, 2017, 4(1): 31-35.

[5] Lee M, Pyun S B, Chung J, et al. A Further Step to Develop Patient-Friendly Implementation Strategies for Virtual Reality-Based Rehabilitation in Patients With Acute Stroke[J]. Physical therapy, 2016, 96(10): 1554-1564. 